\title{
Viral-mediated RdCVF and RdCVFL expression protects cone and rod photoreceptors in retinal degeneration
}

\author{
Leah C. Byrne, ${ }^{1}$ Deniz Dalkara, ${ }^{2,3,4}$ Gabriel Luna, ${ }^{5}$ Steven K. Fisher, ${ }^{5}$ Emmanuelle Clérin, ${ }^{2,3,4}$ Jose-Alain Sahel, ${ }^{2,3,4}$ \\ Thierry Léveillard, ${ }^{2,3,4}$ and John G. Flannery ${ }^{1}$ \\ ${ }^{1}$ Helen Wills Neuroscience Institute, University of California Berkeley, Berkeley, California, USA. ${ }^{2}$ INSERM, U968, Paris, France. ${ }^{3}$ UPMC University of Paris 06, UMRS 968, Institut de la Vision, Paris, France. \\ ${ }^{4}$ CNRS, UMR 7210, Paris, France. ${ }^{5}$ Neuroscience Research Institute, Department of Molecular, Cellular, and Developmental Biology, University of California Santa Barbara, Santa Barbara, California, USA.
}

\begin{abstract}
Alternative splicing of nucleoredoxin-like 1 (Nxn/1) results in 2 isoforms of the rod-derived cone viability factor. The truncated form (RdCVF) is a thioredoxin-like protein secreted by rods that promotes cone survival, while the full-length isoform (RdCVFL), which contains a thioredoxin fold, is involved in oxidative signaling and protection against hyperoxia. Here, we evaluated the effects of these different isoforms in 2 murine models of rod-cone dystrophy. We used adenoassociated virus (AAV) to express these isoforms in mice and found that both systemic and intravitreal injection of engineered AAV vectors resulted in RdCVF and RdCVFL expression in the eye. Systemic delivery of AAV92YF vectors in neonates resulted in earlier onset of RdCVF and RdCVFL expression compared with that observed with intraocular injection using the same vectors at P14. We also evaluated the efficacy of intravitreal injection using a recently developed photoreceptor-transducing AAV variant $(7 \mathrm{m8})$ at P14. Systemic administration of AAV92YF-RdCVF improved cone function and delayed cone loss, while AAV92YF-RdCVFL increased rhodopsin mRNA and reduced oxidative stress by-products. Intravitreal 7m8-RdCVF slowed the rate of cone cell death and increased the amplitude of the photopic electroretinogram. Together, these results indicate different functions for $\mathrm{Nxn} / 1$ isoforms in the retina and suggest that RdCVF gene therapy has potential for treating retinal degenerative disease.
\end{abstract}

\section{Introduction}

Rod-cone dystrophies, such as retinitis pigmentosa (RP), are genetically heterogeneous retinal degenerative diseases characterized by the progressive death of rod photoreceptors, followed by the consecutive loss of cones. RP is one of the most common forms of inherited retinal degeneration, affecting approximately 1 in 3,500 people worldwide (1). Over 40 mutations causing $\mathrm{RP}$ have been identified to date (2), with the majority of these mutations occurring in rod-specific transcripts. RP patients initially present with loss of vision under dim-light conditions as a result of rod dysfunction, with relative preservation of macular cone-mediated vision. As the disease progresses, however, the primary loss of rods is followed by cone degeneration and a deficit in corresponding cone-mediated vision. In modern society, in which much of the environment is artificially lit and many activities rely on high-acuity color vision, retention of cone-mediated sight in RP patients would lead to a significant improvement in their quality of life.

The loss of cones in RP subsets caused by rod-specific mutations is poorly understood, although several mechanisms that are not necessarily mutually exclusive have been proposed. Several hypothesized mechanisms implicate a "neighbor effect," whereby cone death is a consequence of the release of endotoxins from the

Authorship note: Thierry Léveillard and John G. Flannery contributed equally to this work. Conflict of interest: Thierry Léveillard and Jose-Alain Sahel are patent holders on the use of RdCVF for the treatment of neurological disease.

Submitted: August 7, 2014; Accepted: October 23, 2014.

Reference information: J Clin Invest. 2015;125(1):105-116. doi:10.1172/JCI65654. degeneration of surrounding rods, or occurs as a result of the loss of contact with rods, retinal pigment epithelium (RPE), or Müller glia. Alternatively, activation of Müller cells and the release of toxic molecules may play a role. Another hypothesis is that the quantities of oxygen or retinoids delivered to the photoreceptor layer by the RPE from the choroidal blood circulation become excessive and toxic as the metabolic load of rods is lost (3). Punzo et al. showed evidence that in murine models of retinal degeneration, cones die in part as a result of starvation and nutritional imbalance, driven by the insulin/mammalian target of rapamycin pathway (4). Additionally, it has been suggested that the loss of a survival factor secreted by rods and required for cone survival contributes to cone loss $(5,6)$.

In agreement with the last hypothesis, transplanted healthy retinal tissue has been shown to support cone survival in areas distant from the grafted tissue in the $r d 1$ mouse $(7,8)$. The rod-derived cone viability factor (RdCVF) was originally identified from a high-throughput method of screening cDNA libraries as a candidate molecule responsible for this rescue effect (5). Rods secrete RdCVF, and, therefore, as rods die, the source of this paracrine factor is lost and RdCVF levels decrease. The loss of expression of RdCVF, and secreted factors like it, may therefore contribute to the secondary wave of cone degeneration observed in rod-cone dystrophies. RdCVF has been shown to mediate cone survival both in culture (9) and when injected subretinally into mouse and rat models of recessive and dominant forms of RP $(5,10)$. Disruption of Nxnl1, the gene encoding RdCVF, renders mouse photoreceptors increasingly susceptible to photoreceptor dysfunction and cone loss over time (11). 
Nxnl1 codes for 2 isoforms of RdCVF through differential splicing. The isoform mediating cone survival is a truncated form of its longer counterpart, RdCVFL, which includes a C-terminal extension conferring enzymatic function (12). RdCVFL, which contains all the amino acids of RdCVF, is encoded by exons 1 and 2 of the Nxnl1 gene and is a member of the thioredoxin family (13). Thioredoxins have diverse functions, including maintaining the proper reducing environment in cells and participating in apoptotic pathways. These functions are accomplished via thiol oxidoreductase reactions mediated by a conserved CXXC catalytic site within a thioredoxin fold (14).

The exact mechanisms by which these 2 isoforms function in the retina remain unclear. Here, we describe experiments that study the bifunctional nature of the Nxnl1 gene by evaluating the effects of expression of the 2 RdCVF isoforms RdCVF and RdCVFL via gene transfer in the $r d 10$ and $\mathrm{P} 23 \mathrm{H}$ mouse models of rod-cone dystrophy. The $r d 10$ mouse is a well-characterized model of retinal degeneration resulting from a mutation in the $\beta$ subunit of PDE6, the rod-specific cyclic GMP phosphodiesterase $(15,16)$. rd10 retinal degeneration is slower than the rate of degeneration in the most widely studied model of recessive retinal degeneration, the rd1 mouse, which loses the majority of photoreceptors between P15 and P20. In $r d 10$ mice, rod loss begins at P18 and peaks at approximately P25, so that the major phase of rod loss does not overlap with the terminal differentiation of photoreceptors (17). The $r d 10$ mouse model is amenable to gene therapy $(18,19)$, and antioxidant treatments have been shown to slow rod loss in this mouse model (20). In addition, rearing in dim-light conditions has been shown to slow the rate of retinal degeneration, extending the window of opportunity for therapeutic treatment (18).

In contrast, the $\mathrm{P} 23 \mathrm{H}$ mouse is a dominant model of RP that is generated by knock-in of a mouse opsin gene carrying the $\mathrm{P} 23 \mathrm{H}$ mutation (21). In humans, the $\mathrm{P} 23 \mathrm{H}$ mutation is the most frequent opsin mutation associated with autosomal dominant RP, comprising about $10 \%$ of patients (22). Homozygous $\mathrm{P} 23 \mathrm{H}$ mice lose rod photoreceptors quickly, and by P63, most photoreceptor cells are lost. Heterozygous $\mathrm{P} 23 \mathrm{H} /+$ mice lose rods more slowly, with 2 rows of cells remaining in the ventral outer nuclear layer (ONL) and 4 rows in the dorsal ONL at P112.

Here, we investigate the effects of AAV-mediated expression of RdCVF and RdCVFL. These studies use 2 routes of viral vector administration: (a) systemic injection of AAV92YF, a variant of AAV9, via the tail vein at P1 and (b) intravitreal injection at P15. Early systemic injection allows for the onset of expression of the transgene encoded by the AAV vector at an early time point in the course of degeneration, before the major phase of rod cell death and safely within the window of opportunity for evaluating the effect of the transgene expression on rod and cone degeneration. Systemic delivery is not yet a clinically relevant mode of gene delivery to the retina, however, since many other tissues are simultaneously infected, and the immune responses represent a major barrier to this approach. Therefore, we also investigated the effects of intravitreal injection (a commonly used technique for intraocular delivery) of a novel variant of AAV called $7 \mathrm{~m} 8$, which transduces photoreceptors from the vitreous (23).

We show here that expression of the 2 isoforms of RdCVF has positive, contrasting effects on rod and cone survival. Increased expression of RdCVF via systemic and intravitreal injections led to structural and functional rescue of cone photoreceptors in both $r d 10$ and $\mathrm{P} 23 \mathrm{H}$ mouse models, but had little effect on rods. We found that RdCVFL on its own did not significantly rescue cones, although coexpression of RdCVF and RdCVFL increased the observed rescue effect in $r d 10$ mice. In contrast, expression of RdCVFL early in the course of the disease in dark-reared $r d 10$ animals prolonged rod function, increased levels of rhodopsin, and decreased the by-products of cellular oxidative stress.

These results indicate that RdCVF and RdCVFL protect photoreceptors through separate complementary mechanisms and show proof of concept for a widely applicable viral vectormediated gene therapy that may be able to prolong vision, independently of the underlying mutation, in patients suffering from a variety of rod-cone dystrophies.

\section{Results}

Systemic delivery of AAV92YF via tail-vein injections at P1. We examined the therapeutic effects of early RdCVF and RdCVFL delivery by i.v. tail-vein injection of a self-complementary AAV9 vector with 2 tyrosine-to-phenylalanine mutations (AAV92YF). Intraocular injections into the developing eye are impractical in the initial postnatal week; however, AAV92YF has been shown to cross the blood-retina barrier when injected into the tail vein at P1, leading to high levels of gene expression across the retina (24). Systemic delivery leads to onset of expression in the retina significantly earlier in development, before substantial numbers of rods are lost. AAV92YF with a ubiquitous CAG promoter driving expression of GFP (AAV92YF-scCAG-GFP) resulted in early retinal expression, which was visible in retinal flat mounts at P8 (Supplemental Figure 1A; supplemental material available online with this article; doi:10.1172/JCI65654DS1) and by in vivo fundus imaging immediately after eye opening at P15 (Figure 1A). Retinal flat mounts revealed that large numbers of photoreceptors were transduced (Figure 1B). At P35, we observed GFP expression in all retinal layers, in ganglion cells, Müller glia, amacrine cells, and photoreceptors, as well as in the RPE. We observed a similar pattern of widespread and strong expression, which was easily visible without immunolabeling, in WT (Figure 1C) and $r d 10$ (Figure 1D) retinae. Quantitative reverse transcriptase PCR (qRT-PCR) performed on mRNA collected from $r d 10$ mice raised in a normal light/dark cycle and injected at P1 with AAV92YF-scCAG-RdCVF, AAV92YF-scCAG-RdCVFL, or PBS revealed that i.v. injection of the virus resulted in high levels of RdCVF expression in the retina at P35 (Figure 1E). As expected, Nxnl1 mRNA levels were greatly reduced in PBS-injected $r d 10$ animals compared with levels in WT animals of the same age ( $5 \% \pm 5 \%$ of WT levels). In contrast, expression levels after AAV-mediated gene delivery were comparable to those of endogenous Nxnl1 levels in WT animals (AAV92YF-RdCVF $=79 \% \pm 30 \%$ of WT levels; AAV92YF-RdCVFL $=59 \% \pm$ $13 \%$ of WT levels). Rhodopsin mRNA expression levels remained low across conditions, indicating similar rates of rod photoreceptor loss in treated and untreated animals at P35 (AAV92YF-RdCVF $=8 \%$ $\pm 3 \%$ of WT levels; AAV92YF-RdCVFL $=1 \% \pm 1 \%$ of WT levels; PBS $6 \% \pm 1 \%$ of WT levels).

Transgene expression from AAV-mediated delivery is dose dependent (Supplemental Figure 2). Animals injected with $2 \mathrm{E}+11$, $5 \mathrm{E}+11$, or $1 \mathrm{E}+12$ viral particles of AAV92YF-scCAG-GFP and 

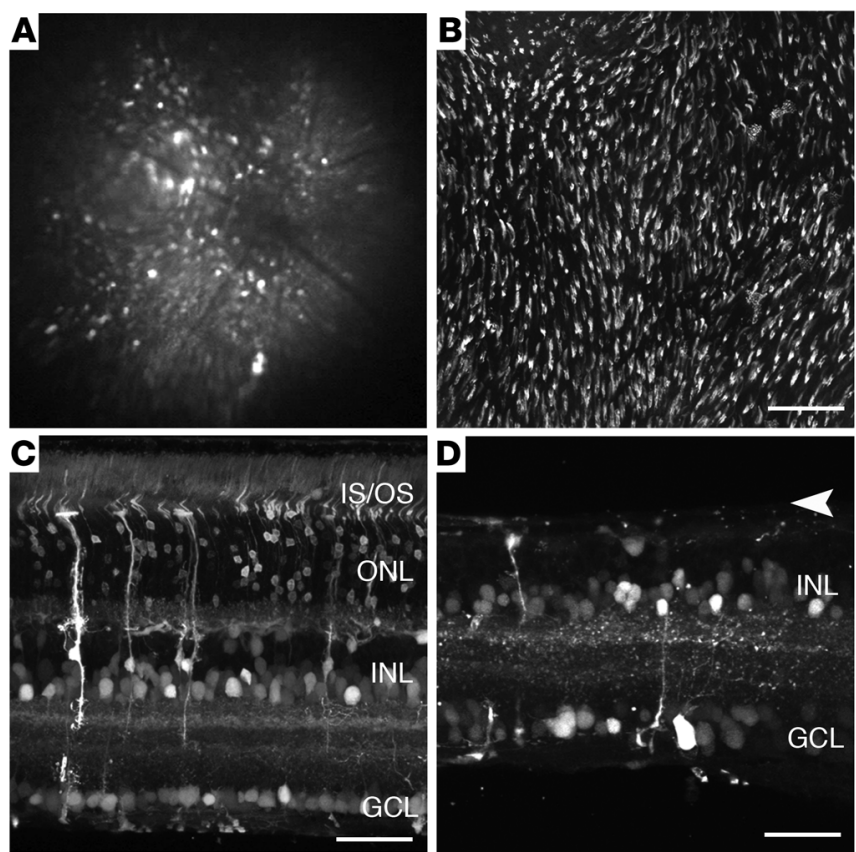

\section{$\mathbf{E}$}

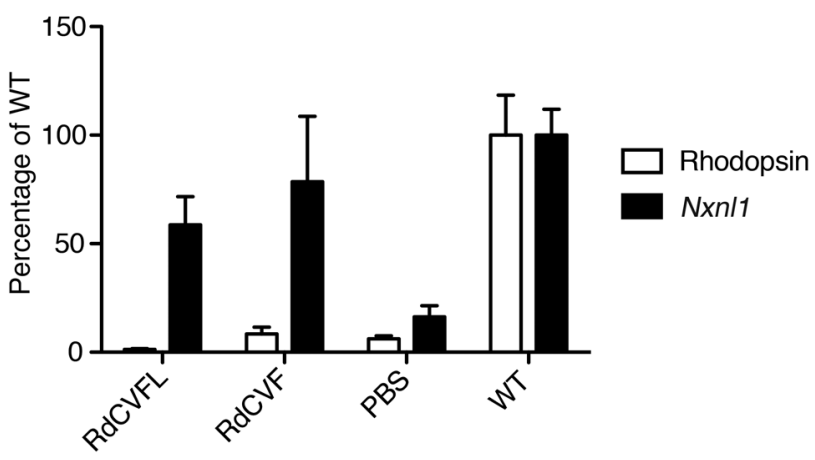

imaged 1 month after injection showed that GFP levels increased with higher viral titers (Supplemental Figure 2A). Additionally, qRT-PCR from animals injected with a range of AAV92YF-scCAG-RdCVF titers showed that RdCVF expression levels increased with the injection of higher numbers of viral particles (Supplemental Figure 2B). qRT-PCR revealed that 4 months after treatment, injection of $\mathrm{E}+11$ particles resulted in RdCVF levels of $11 \% \pm 2 \%$ of those in WT animals, while injection of $\mathrm{E}+12$ particles resulted in RdCVF levels of $68 \% \pm 16 \%$ of WT levels.

Effect of injection of AAV92YF-scCAG-RdCVF on cone function. Mice were injected i.v. with AAV92YF-scCAG-RdCVF at P1 and subsequently raised in a normal 12-hour light/12-hour dark cycle. We then measured photopic electroretinograms (ERGs) to determine the effect of RdCVF expression on cone function. Representative ERG traces illustrated improved waveforms and amplitudes in the ERGs recorded from AAV92YF-scCAG-RdCVF-injected eyes compared with the ERGs of mice injected with AAV92YF-scCAG-RdCVFL, AAV92YF-scCAG-GFP, or PBS (Figure 2A). AAV92YF-scCAG-mediated expression of RdCVF resulted in significantly higher amplitudes of photopic ERG b-waves $(97.1 \pm 10.67 \mu \mathrm{V})$ compared with those measured in animals injected with AAV92YF-scCAG-RdCVFL $(46.7 \pm 6.4 \mu \mathrm{V}$, $P<0.005)$, AAV92YF-scCAG-GFP $(46.6 \pm 14.9 \mu \mathrm{V}, P<0.01)$, or
Figure 1. Expression profile of AAV92YF in the retina after systemic delivery at P1. (A) Injection of AAV92YF-scCAG-GFP resulted in strong pan-retinal GFP expression, as shown in a fundus image of a P45 WT mouse. (B) Retinal flat mount showing large numbers of GFP-expressing photoreceptors in the central retina of a WT mouse 45 days after injection. (C) AAV92YF transduced cells in the GCL, INL, and ONL in a WT mouse. (D) Injection of AAV92YF led to gene expression across all layers of the retina in a P45 rd10 mouse. The ONL was significantly reduced at this time point (arrowhead). (E) qRT-PCR showing high levels of Nxn/1 mRNA from eyes injected with AAV92YF-scCAG-RdCVF or AAV92YF-scCAG-RdCVFL. PBS-injected animals had markedly reduced levels of Nxn/1 mRNA. Levels of rhodopsin expression were low across conditions in rd10 eyes. Eyes were collected at P35 from 3 animals injected in the tail vein at P1 and raised in a normal light/dark cycle. One eye was taken from each animal, and opposite eyes were used for the cone labeling and quantification shown in Figure 6. Scale bars: $40 \mu \mathrm{m}$ (B); $50 \mu \mathrm{m}$ (C and D).

PBS (56.5 $\pm 4.64 \mu \mathrm{V}, P<0.01)$ (Figure 2B). WT b-wave amplitudes were $156.6 \pm 11.4 \mu \mathrm{V}$. (ERGs were recorded from 5 animals for each condition.) We also recorded photopic flicker ERGs as a measure of cone function. Representative flicker ERG traces illustrated improved amplitudes and waveforms compared with those measured in GFP-injected animals (Figure 2C). Coadministration of AAV92YF-RdCVF and AAV92YF-RdCVFL did not significantly increase photopic ERG amplitudes compared with those measured in animals receiving AAV92YF-RdCVF administration alone (photopic ERG amplitudes in coinjected eyes were $92.71 \pm 11.71 \mu \mathrm{V})$.

We found that rescue of the photopic ERG was also dose dependent (Figure 3), indicating that a minimum level of RdCVF expression must be achieved for significant rescue to occur. ERG amplitudes were only slightly increased in $r d 10$ mice following systemic injection of $\mathrm{E}+11$ viral genomes, while injection of $\mathrm{E}+12$ viral genomes resulted in markedly higher ERG amplitudes. Representative photopic ERG traces $(n=3)$ illustrated a higher level of rescue following injection of greater numbers of viral particles.

Transgene expression following injection of AAV92YF is also long lasting. GFP expression in AAV9-scCAG-GFP-injected animals was readily visible in retinal flat mounts imaged 1 year after injection (Supplemental Figure 3A). In $r d 10$ animals injected with AAV92YF-scCAG-RdCVF, RT-PCR revealed elevated levels of RdCVF 1 year after injection (Supplemental Figure 3B).

Cone densities in animals injected with AAV92YF-scCAG-Rd$C V F$. Automated counting of immunofluorescence-labeled cone outer segments was used to quantify cone survival. Entire retinae from animals previously used for ERG recordings were flat mounted and stained with antibodies against S-opsin and M/L-opsin. High-resolution $(\times 40) z$-stack images were collected across the entire retina and were registered and stitched together to create mosaics. Mosaics of retinal flat mounts revealed higher numbers of cones labeled with both S- and M/L-opsin in whole flat mounts from animals injected with AAV92YF-scCAG-RdCVF (Figure 4A). Higher numbers of surviving cones of both types were apparent in higher-resolution images near the optic nerve head, the region of the retina with the most severe degeneration (Figure $4 \mathrm{~B}$ ). Automated quantification of cone densities in AAV92YF-scCAG-RdCVF and PBS-injected retinae revealed significantly higher numbers of both S- and $\mathrm{M} / \mathrm{L}$-cones per $\mathrm{mm}^{2}$ in treated eyes 

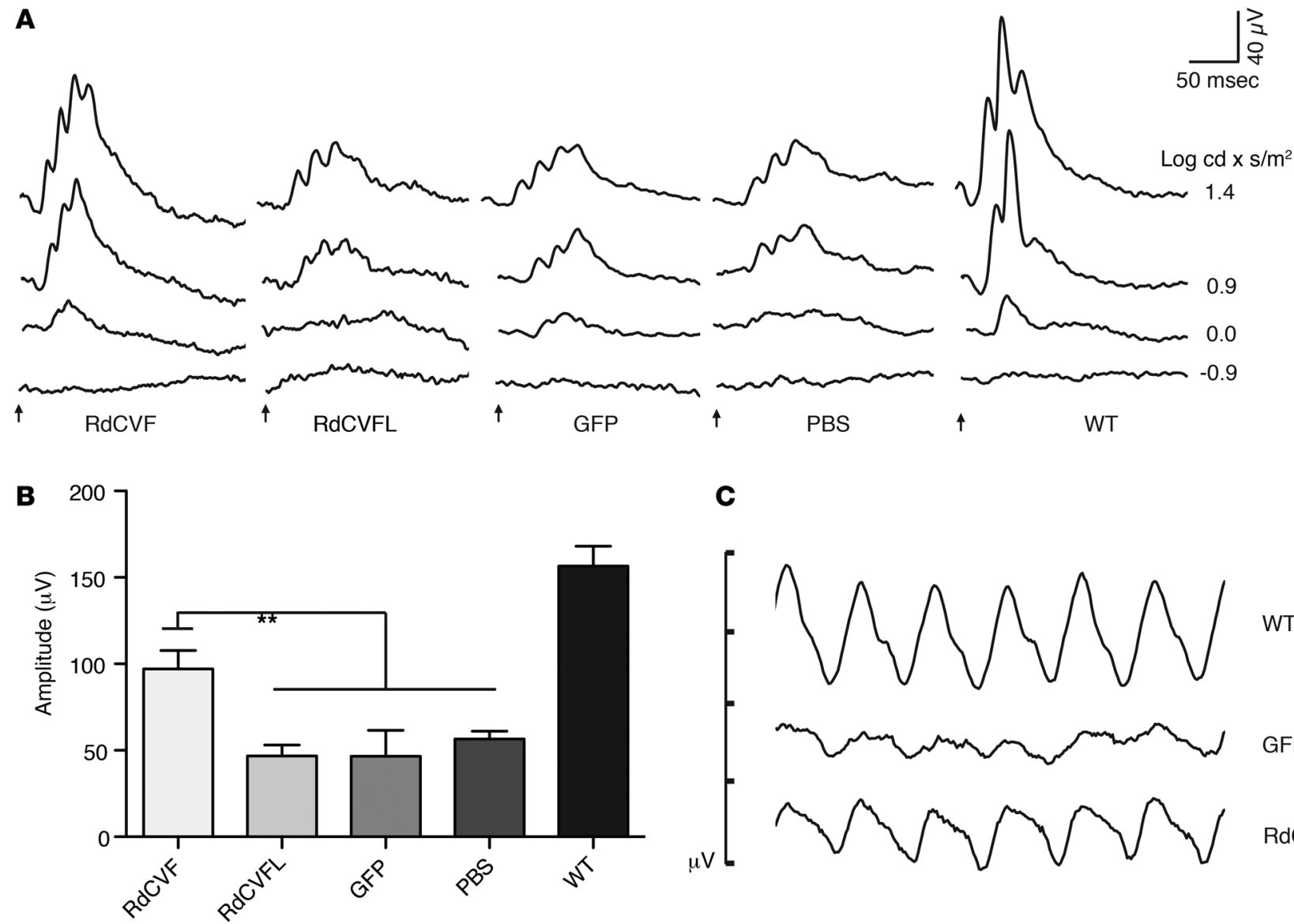

\section{C}

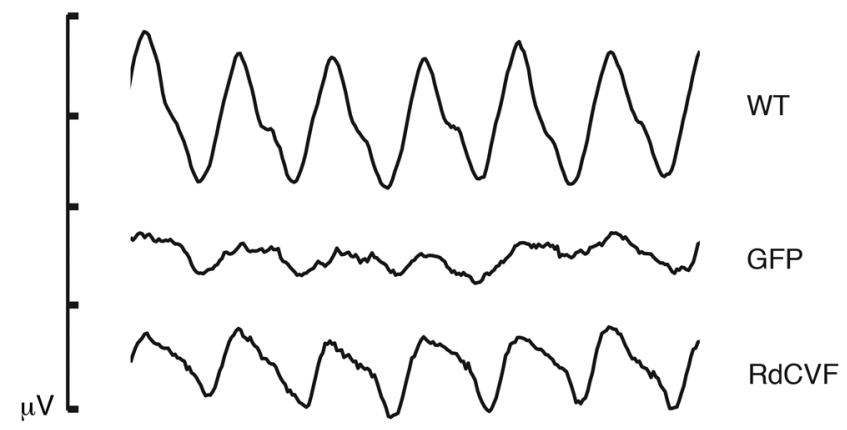

Figure 2. Early delivery of RdCVF leads to sustained photopic ERG amplitudes. (A) Photopic b-wave amplitudes were significantly higher in animals injected with AAV92YF-scCAG-RdCVF compared with those in mice injected with AAV92YF-scCAG-RdCVFL, AAV92YF-scCAG-GFP, or PBS. (B) Quantification $(n=5)$ of photopic ERG traces presented in $\mathbf{A} .{ }^{*} P<0.01$. Data are presented as the mean \pm SD, and significance was determined using a 1-way ANOVA with Tukey's post-hoc multiple comparisons test. (C) Representative flicker ERGs recorded from AAV92YF-scCAG-CFP-injected, AAV92YF-scCAG-RdCVFinjected, or WT mice $(n=5)$ illustrate improved amplitudes and more normal waveforms in treated animals. Marks on scale bar indicate 5 - $\mu \mathrm{V}$ increments.

compared with those observed in PBS-injected eyes. S-cone densities were: RdCVF-treated eyes: $5,573 \pm 211 / \mathrm{mm}^{2}$; PBS-treated eyes: $2,961 \pm 917 / \mathrm{mm}^{2} ; P<0.01$, WT eyes: 7,446 $\pm 868 / \mathrm{mm}^{2}$. M/Lcone densities were: RdCVF-treated eyes: $8,755 \pm 1,572 / \mathrm{mm}^{2}$; PBS-treated eyes: 2,682 $\pm 293 / \mathrm{mm}^{2} ; P<0.01$; WT eyes: 9,761 \pm $784 / \mathrm{mm}^{2}$ (Figure $4 \mathrm{C}$ ).

Effect of systemic injection of AAV92YF-scCAG-RdCVFL. Animals were raised in the dark to slow the rate of rod loss and allow for onset of RdCVFL expression in rods before apoptosis. Reducing light exposure slowed the rate of photoreceptor degeneration as shown previously (18). Mice were injected at P1 with AAV92YF-scCAG-RdCVFL, AAV92YF-scCAG-GFP, or PBS ( $n=6$ each group). We recorded scotopic full-field ERGs on a weekly basis. Recordings from the highest-intensity light stimulus made 3, 4, and 5 weeks after injection revealed a smaller loss of a-wave amplitudes at weeks 3 and 4, but this amelioration was no longer apparent at 5 weeks (Figure 5A). The difference was statistically significant $(P<0.05)$ only at 4 weeks after injection. We performed a more detailed analysis on a second litter of mice injected with AAV92YF-scCAG-RdCVFL or PBS (Figure 5B). In this group, 4 weeks after injection, we noted the most significant differences in a-wave amplitudes at lower light intensities $\left(-1\right.$ and $\left.-2 \log \mathrm{cd} \times \mathrm{s} / \mathrm{m}^{2}\right)$. Representative ERG traces illustrated the preservation of a-wave and b-wave amplitudes in animals injected with AAV92YF-scCAG-RdCVFL compared with those in control animals injected with GFP or PBS (Figure 5C). Photopic ERG recordings revealed a delay in the decrease of the cone ERGs in RdCVFL-expressing eyes that was most noticeable 5 weeks after injection, but this difference was not statistically significant.

qRT-PCR on rhodopsin $m R N A$ in dark-reared animals injected systemically with AAV92YF-scCAG-RdCVFL. qRT-PCR performed on mRNA collected from P28 animals injected with AAV92YF-RdCVFL, GFP, or RdCVF at P1 and raised in complete darkness revealed an increase in rhodopsin mRNA levels in animals injected with AAV92YF-RdCVFL $(82 \% \pm 21 \%$ of WT, $P<0.05)$, but not in those injected with PBS ( $56 \% \pm 6 \%$ of WT), AAV92YF-GFP $(59 \%$ $\pm 15 \%$ of WT), or AAV92YF-RdCVF ( $57 \% \pm 16 \%$ of WT) (Figure $5 \mathrm{D})$. These results, together with the rhodopsin mRNA levels measured in animals raised in a normal light/dark cycle, indicate the importance of RdCVFL delivery during a short window of opportunity for the effects of expression to be observed in this relatively rapid model of retinal degeneration, which begins to lose photoreceptors in the first few weeks of life, as we observed no effect on rhodopsin mRNA levels in animals injected with AAV92YF-RdCVFL and raised in the light (Figure 1E). 


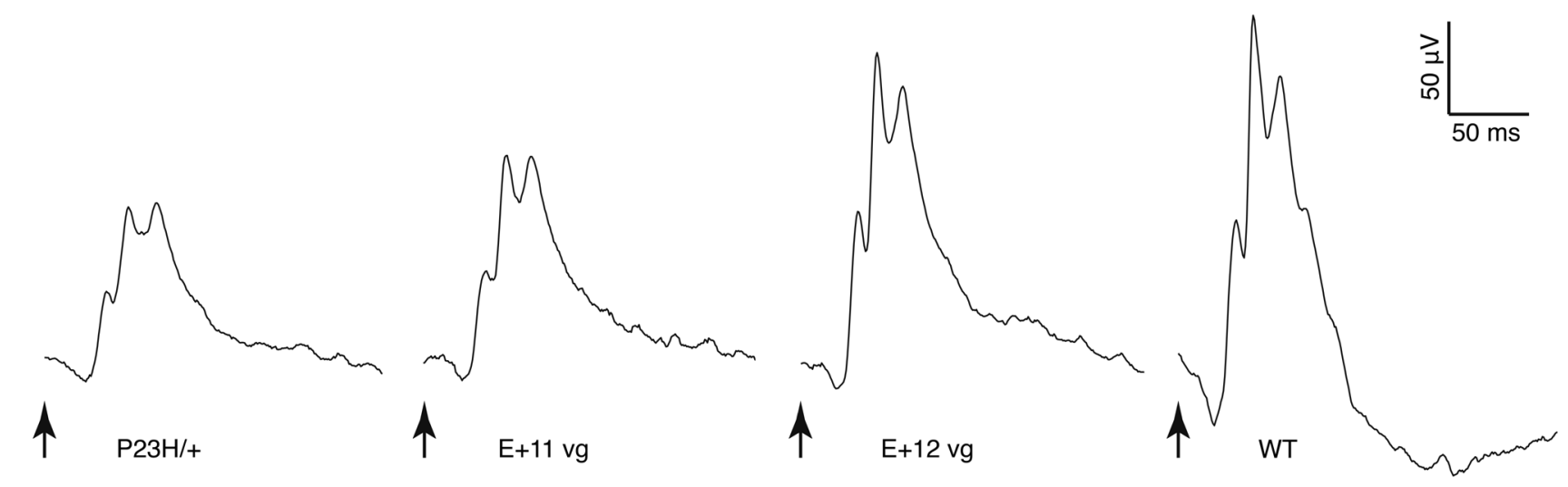

Figure 3. Rescue of ERG amplitudes is dose dependent. Representative traces $(n=3)$ are photopic ERGs from $r d 10$ mice 1 month after injection with E+11 or $\mathrm{E}+12$ particles. Arrows indicate the onset of the light flash.

Measurement of lipid peroxidation. We performed a thiobarbituric acid reactive substances (TBARS) assay to determine levels of the lipid peroxidation by-product malondialdehyde (MDA) in retinae treated with AAV92YF-scCAG-RdCVFL, AAV92YF-scCAG-RdCVF, AAV92YF-scCAG-GFP, or PBS. The test was performed on 3 pooled retinae and repeated 3 times. A representative assay is shown in Figure 5E. We observed that MDA levels were decreased by $18 \% \pm 0.9 \%$ in RdCVFL-treated eyes compared with those in untreated eyes.

Intravitreal injection of the novel viral variant $7 \mathrm{~m} 8$. We characterized the viral tropism and expression pattern of the novel viral variant $7 \mathrm{~m} 8$ in WT and $r d 10$ mice after intravitreal injection at $\mathrm{P} 15.7 \mathrm{~m} 8$ is a variant of AAV2 developed to transduce the outer retina following intravitreal injection (23). Importantly, this variant transduces photoreceptors without subretinal injection, which has been shown to cause injury response and release of trophic factors (25). Intravitreal injections of a self-complementary $7 \mathrm{~m} 8$ vector encoding GFP at P15 resulted in strong expression by 1 week after injection (Supplemental Figure 1B), which was also clearly visible in fundus images at P45 (Figure 6A). Flat-mounted retinae showed that large numbers of photoreceptors were transduced by $7 \mathrm{~m} 8$ (Figure 6B). Confocal imaging of retinal cross sections revealed GFP expression in cells lying in the ganglion cell layer (GCL), inner nuclear layer (INL), and ONL in WT (Figure 6C) and $r d 10$ mice (Figure 6D; arrowhead indicates loss of photoreceptors in the ONL).

RdCVF expression levels following injection of $7 \mathrm{~m} 8$ were evaluated by qRT-PCR (Figure 6E). mRNA levels in P45 rd10 mice injected with the viral vectors encoding RdCVF, RdCVFL, or GFP were quantified. As expected, levels of RdCVF mRNA were reduced in control GFP-injected $r d 10$ mice compared with those in WT mice $(4 \% \pm 1 \%$ in WT). Intraocular injection of $7 \mathrm{~m} 8$-scCAG-RdCVF or $7 \mathrm{~m} 8$-scCAG-RdCVFL at P14 resulted in higher levels of Nxnl1 mRNA. Levels were $127 \% \pm 35 \%$ of those in WT mice in animals injected with $7 \mathrm{~m} 8$-scCAG-RdCVF and $91 \%$ $\pm 10 \%$ of WT levels in $7 \mathrm{~m} 8$-scCAG-RdCVFL-injected animals. We found that rhodopsin levels were uniformly low in all retinae measured $(\mathrm{RdCVF}=3 \% \pm 1 \%$ of WT; RdCVFL $=1 \% \pm 1 \%$ of WT; $\mathrm{PBS}=1 \% \pm 1 \%$ of $\mathrm{WT})$.
Effects of expression of RdCVF and RdCVFL on cone structure and function. We next examined the effect of intraocular injection of $7 \mathrm{~m} 8$ encoding RdCVF and RdCVFL on cone rescue in dark-reared rd10 mice (Figure 7). Injection of 7m8-scCAG-RdCVFL at P14 resulted in a small, statistically insignificant increase in photopic ERG b-wave amplitudes $(74 \pm 4.8 \mu \mathrm{V})$ compared with the amplitudes measured in PBS- $(65 \pm 5 \mu \mathrm{V})$ and GFP-injected $(69 \pm 7.3 \mu \mathrm{V})$ eyes (Figure 7A). Injection of $7 \mathrm{~m} 8$-scCAG-RdCVF significantly increased ERG b-wave amplitudes $(89 \pm 7.9 \mu \mathrm{V}, P<0.05)$ compared with those in GFP- or PBS-injected eyes. We found that coinjection of 7m8-scCAG-RdCVF and 7m8-scCAG-RdCVFL resulted in greater rescue of the photopic ERG, which was 53\% higher than that in untreated eyes $(99.75 \pm 5.7 \mu \mathrm{V}, P<0.01)$ and $17 \%$ higher than that in RdCVF-treated eyes alone $(P<0.05)$. Under the conditions tested, injection of $7 \mathrm{~m} 8$-RdCVF and $7 \mathrm{~m} 8$-RdCVFL did not result in any statistically significant change in scotopic ERG amplitudes. In all animals, 1 eye was injected with $7 \mathrm{~m} 8$-scCAG-GFP or PBS as an internal control. Representative ERG traces illustrated increased amplitudes in eyes expressing RdCVF or RdCVF plus RdCVFL for the photopic b-wave (Figure 7B) and in flicker ERGs recorded at $30 \mathrm{~Hz}$ (Figure $7 \mathrm{C}$ ).

Cone labeling in eyes injected with $7 m 8-s c C A G-R d C V F$. Anti-S-opsin and anti-M/L-opsin antibodies were used to label cone populations in 7m8-scCAG-RdCVF- or 7m8-scCAG-GFPinjected eyes, and high-resolution $(\times 40)$ image mosaics were created (Figure 7D). Labeling revealed increased numbers of cones, most notably in central areas of the retina, near the optic nerve head (asterisk in Figure 7D, lower panels). Automated quantification of labeled cones across the entire retina revealed an increase in cones expressing S- and M/L-opsin in 7m8-scCAG-RdCVFexpressing eyes (S-cones: 1,644 $\pm 436, P<0.05$; M/L-cones: $2,205 \pm 264)$ compared with the number of cones in contralateral 7m8-scCAG-GFP-injected eyes (S-cones: 1,254 \pm 326 ; $\mathrm{M} / \mathrm{L}$-cones $1,112 \pm 419 ; P<0.05$ ) (Figure 7E).

Effects of expression of RdCVF and RdCVFL in the P23H mouse. $7 \mathrm{~m} 8$ was next used to deliver RdCVF or RdCVFL in the homozygous (Figure $8 \mathrm{~A}$ ) or heterozygous (Figure $8 \mathrm{~B}$ ) P23H mouse, a model of dominant RP (21). Quantification of ERG recordings showed that in homozygous $\mathrm{P} 23 \mathrm{H} / \mathrm{P} 23 \mathrm{H}$ mice, injection of 7m8-scCAG-RdCVF 

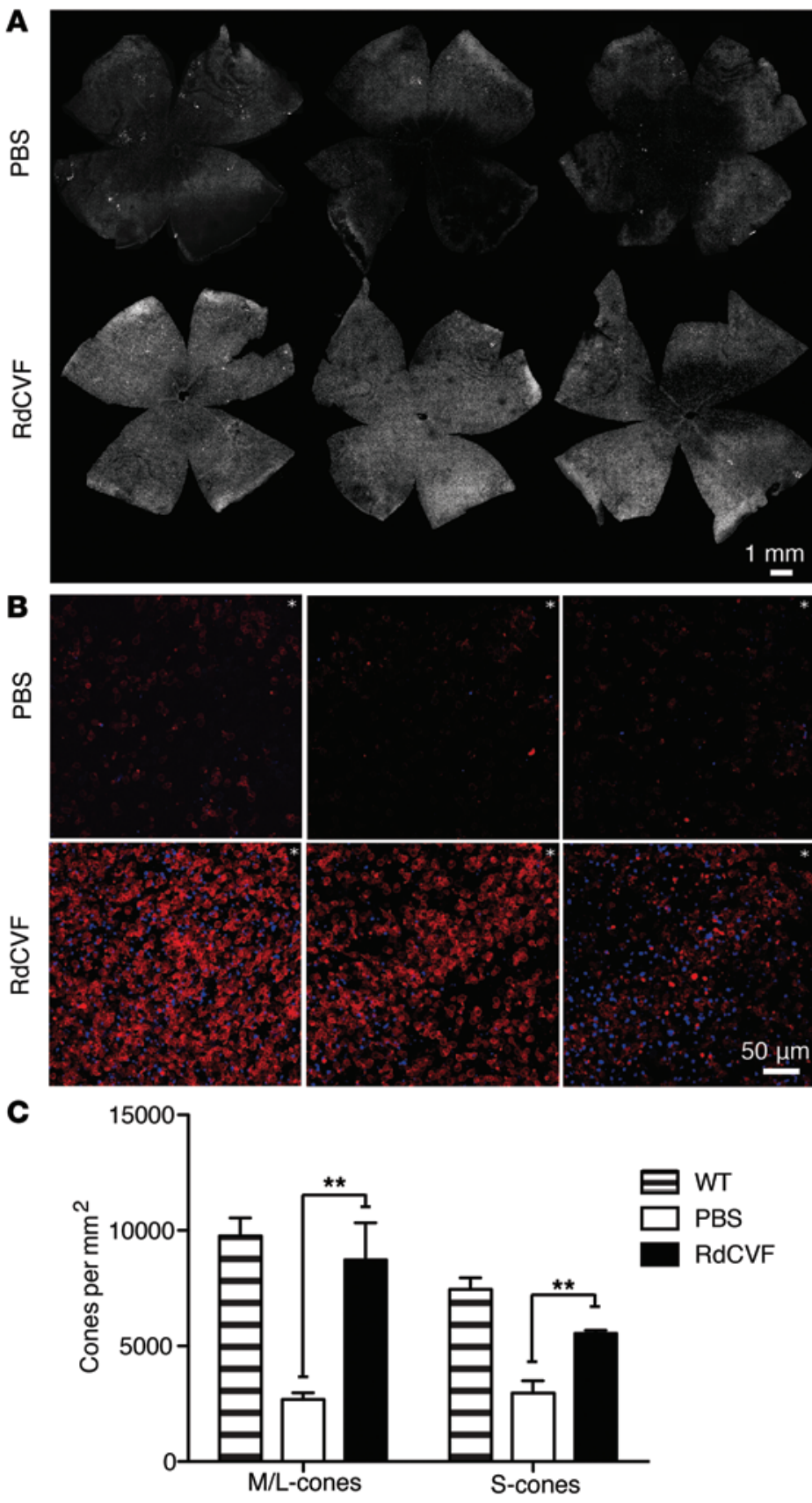

resulted in an increase in photopic ERG amplitudes compared with those recorded in PBS-injected eyes (RdCVF: $39 \pm 15.7 \mu V$; PBS: 19 $\pm 11.3 \mu \mathrm{V} ; P<0.01$ by 2 -tailed, paired Student's $t$ test) 1 month after injection at $\mathrm{P} 8$. In heterozygous $\mathrm{P} 23 \mathrm{H} /+$ mice, which degenerate more slowly and may more closely model human disease, injection of $7 \mathrm{~m} 8$-scCAG-RdCVF at P21 resulted in an increase in photopic ERG recording amplitudes up to 4 months after treatment compared with those in control GFP-treated contralateral eyes (Figure 8B). (One month after injection, RdCVF: $175 \pm 21.4 \mu \mathrm{V}$ vs. PBS: 107 $\pm 17.2 \mu \mathrm{V} ; 4$ months after injection, RdCVF: $71.5 \pm 18 \mu \mathrm{V}$ vs. PBS: $45 \pm$ $15.6 \mu \mathrm{V} ; 6$ months after injection, RdCVF: $23.8 \pm 14.9 \mu \mathrm{V}$ vs. PBS: 18 $\pm 10.4 \mu \mathrm{V}$.) In contrast, we found that treatment with RdCVFL did not result in any significant change in the amplitudes of photopic ERG recordings at any of the time points measured (results were calculated by repeated-measures 2-way ANOVA with Sidak's mul-
Figure 4. AAV92YF-mediated expression of RdCVF promotes cone survival. (A) Flat mounts of retinae from animals injected with AAV92YF-scCAG-RdCVF and labeled with antibodies against M/L-opsin and S-opsin had greater numbers of cones than did PBS-injected mice. Top row: retinae from PBS-injected mice. Bottom row: retinae from AAV92YF-RdCVF-injected mice. (B) Higher-resolution images near the optic nerve (asterisk, upper right hand corner of each image) show increased density of $\mathrm{M} / \mathrm{L}$-opsin (red) or S-opsin (blue) in the central retina in RdCVF-injected animals. (C) Quantification of cones $(n=3)$ revealed an increase in the density of anti-L-opsin- and anti-S-opsinlabeled cones in retinae expressing RdCVF. Data represent the mean \pm SD. ${ }^{*} P<0.01$ by 2 -tailed, unpaired Student's $t$ test.

tiple comparison's test). (One month after injection, RdCVFL: $135 \pm 47.3 \mu \mathrm{V}$ vs. PBS: $120.25 \pm 53.7 \mu \mathrm{V} ; 4$ months after injection, RdCVFL: $72 \pm 38 \mu \mathrm{V}$ vs. PBS: $72.8 \pm 45.2 \mu \mathrm{V}$; 6 months after injection, RdCVFL: $47.2 \pm 55.8 \mu \mathrm{V}$ vs. PBS: $31 \pm 32 \mu \mathrm{V}$.)

\section{Discussion}

The loss of cones is debilitating for patients afflicted with rod-cone dystrophies such as RP. Much of daily life in modern industrialized societies requires high-acuity color vision, which is mediated by cones, and the loss of these cells in the final stages of retinal degenerative disease severely affects a patient's quality of life. It is estimated that half of the disease-causing genes have been identified for autosomal recessive forms of RP (26). Of those that have been characterized, many have been identified in fewer than 200,000 people, relegating them to orphan status. Creating specific gene replacement therapies for those diseases whose mutations have not been identified and those affecting few people may prove impossible. Therefore, a mutation-independent strategy aimed at supporting the survival of cones is an important goal of translational retinal research.

Here, we demonstrate the efficacy of AAV vectors as a delivery strategy for RdCVF and show that expression of $\mathrm{RdCVF}$ is a promising approach to delaying the loss of cones in patients with rod-cone dystrophy. Viral vectors could potentially be used to express the protein for the life of the patient following a single treatment, and the recent success of clinical trials for Leber congenital amaurosis (LCA) have shown the feasibility and safety of this approach (27-29), indicating that it may be an appropriate method of treatment for many forms of retinal degeneration. AAV-mediated delivery of RdCVF could benefit patients carrying the many rod-specific mutations underlying rod-cone dystrophy, making this therapy a flexible approach to prolonging vision across a spectrum of disorders.

In our study, systemic injection of AAV92YF-scCAG-RdCVF mediated fast onset and high levels of RdCVF expression, leading to greater numbers of surviving cones as well as rescue of cone function as measured by photopic and flicker ERGs against a rodsaturating background. RdCVF expression had little effect on the survival or function of rods, however.

We also studied the activity of RdCVFL in the retina by systemic administration of AAV encoding the long isoform of RdCVF, which contains a C-terminal extension composed of a thioredoxin fold. RdCVFL is a member of the thioredoxin family, and the presence of a thioredoxin fold with enzymatic activity suggests 
A

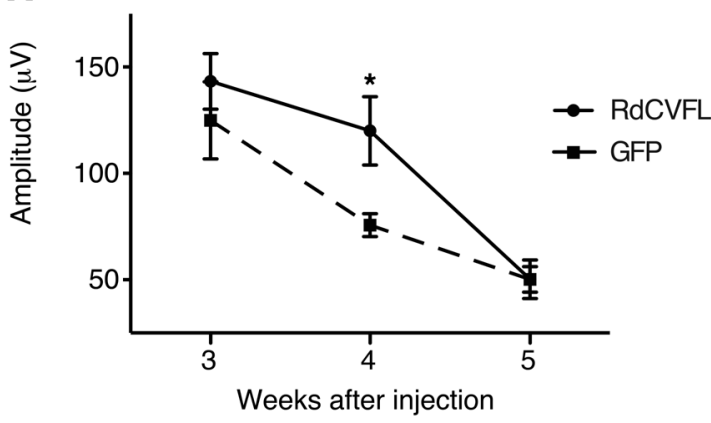

$\mathbf{C}$

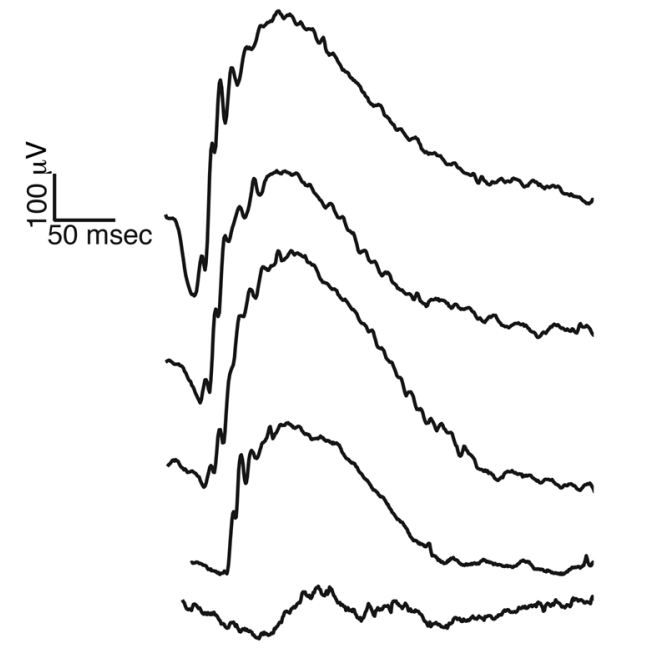

D

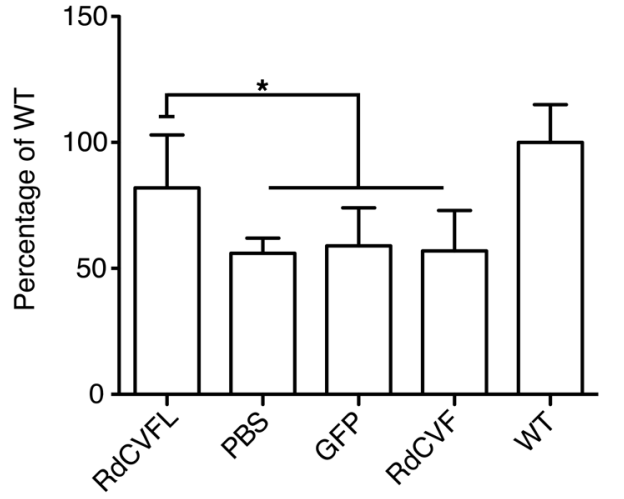

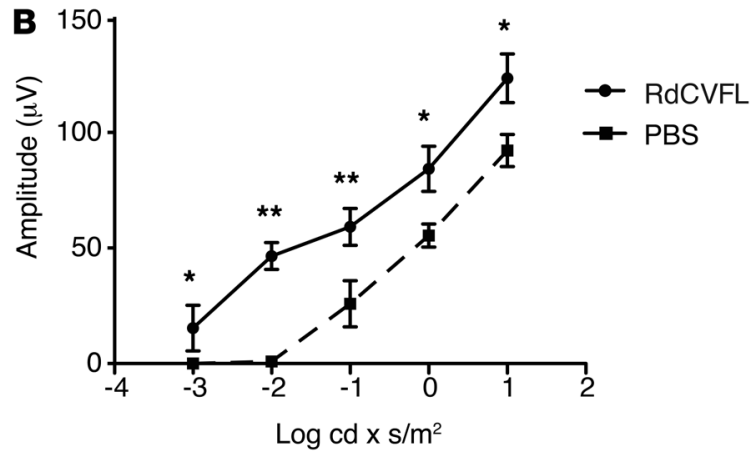

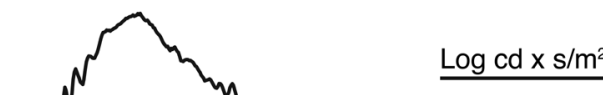

1.0

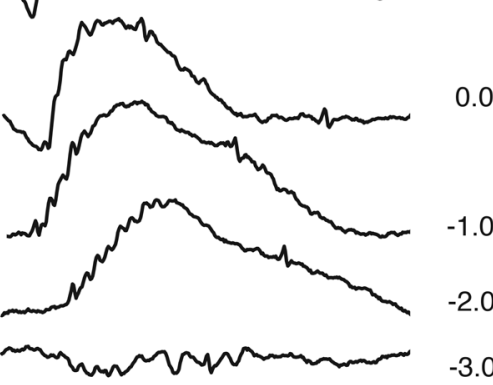

$\mathbf{E}$

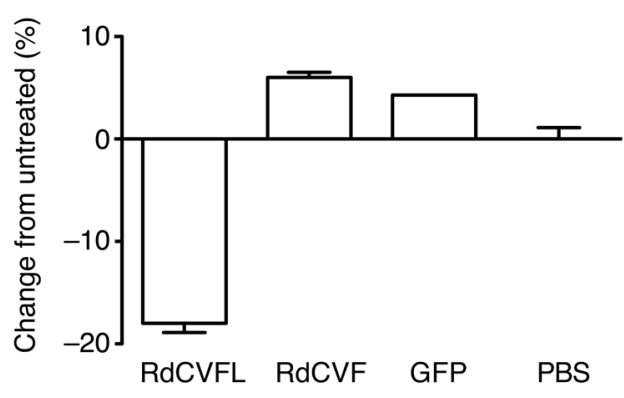

Figure 5. Effects of AAV92YF-mediated delivery of RdCVFL in dark-reared animals. (A) Time course of the amplitude of the full-field scotopic ERG a-wave recorded in dark-reared $r d 10$ mice injected with AAV92YF-RdCVFL $(n=5)$ or AAV92YF-scCAG-CFP $(n=5)$ revealed a delay in loss of retinal responses in treated animals. Four weeks after injection, a significant difference in the amplitude of the ERG a-wave was observed in RdCVFL-injected animals compared with that seen in GFP-injected mice. Five weeks after injection, both groups had progressively reduced a-wave amplitudes, and no difference in ERG amplitudes between the 2 groups was observed. Significance was determined using a repeated-measures 2-way ANOVA with Sidak's multiple comparisons test. (B) A more detailed analysis of ERG traces recorded 4 weeks after injection of AAV92YF-RdCVFL or PBS revealed increased a-wave amplitudes over a range of flash intensities. Significance was determined using a 2-way ANOVA with post-hoc multiple comparisons test. (C) Representative ERG traces from RdCVFL-treated (left traces) or PBS-injected (right traces) animals illustrating increased amplitudes of the scotopic a-wave and b-wave 4 weeks after injection in treated mice. (D) Injection of AAV92YF-scCAG-RdCVFL resulted in an increase in the level of rhodopsin mRNA 4 weeks after injection compared with levels in untreated or GFP- or RdCVF-expressing eyes. Significance was determined by 1-way ANOVA with Tukey's post-hoc multiple comparisons test. (E) TBARS assay revealed reduced levels of MDA, a marker for oxidative stress and lipid peroxidation, in retinae injected with AAV92YF-RdCVFL and collected 4 weeks after injection compared with MDA levels in AAV92YF-RdCVF, AAV92YF-GFP, or PBS-injected eyes ( $n=3$ for all groups). Data are presented as the percentage of change in MDA concentrations compared with those in untreated $r d 10$ eyes \pm SD. ${ }^{*} P<0.05 ;{ }^{* *} P<0.01$.

that RdCVFL has a role similar to that of TRX1 in scavenging free radicals, inhibiting apoptosis, regulating transcription factors, and regulating redox homeostasis (12). The Nxnl1-deficient mouse has disorganized rods that die over time, is prone to damage from hyperoxia, and has increased levels of the lipid peroxi- dation by-product MDA, indicative of oxidative damage to retinal protein (11). In addition, RdCVFL has been shown to interact with the microtubule-binding protein Tau and in vitro to protect against oxidative stress-related phosphorylation of Tau (30). In Alzheimer's disease, hyperphosphorylation of Tau is linked to aggrega- 

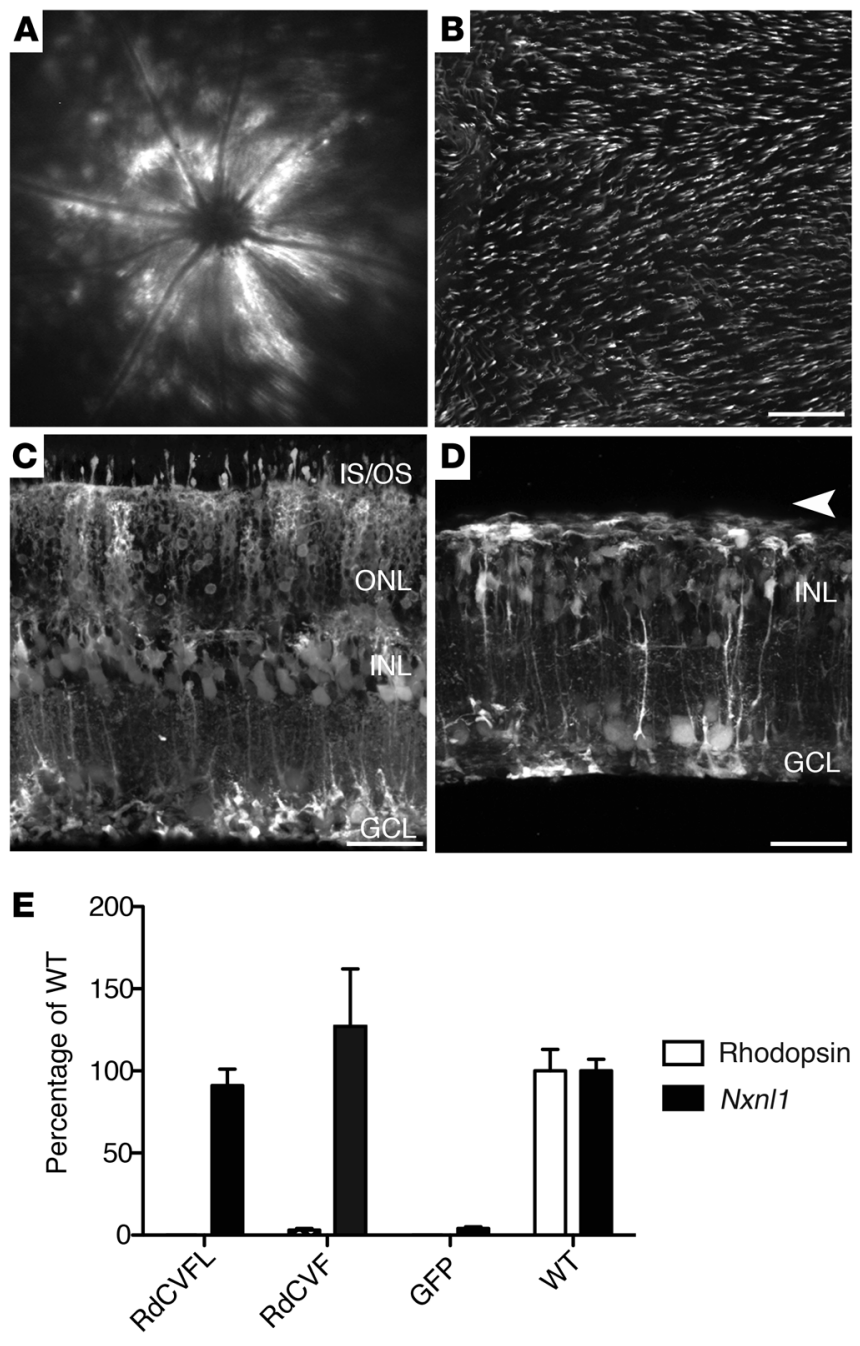

tion of the protein and a decrease in microtubule binding as well as to cytotoxicity. The $\mathrm{Nxnl1}^{-/-}$retina also displays increased Tau phosphorylation and aggregation (11). Together, this evidence suggests that RdCVFL plays a role in protecting against oxidative stress through its activity as a thioredoxin-like enzyme.

Here, we show that early systemic administration of AAV92YF-scCAG-RdCVFL to $r d 10$ mice led to a decrease in MDA levels, supporting the role of RdCVFL as a protein with redox activity. This reduction supports the notion that the increased levels of MDA observed in the $\mathrm{Nxnl1}^{-/-}$mouse are a consequence of the absence of RdCVFL expression in that animal model. In addition, we demonstrate that expression of RdCVFL is followed by a delay in the loss of rod function and that higher levels of rhodopsin mRNA were observed in animals treated with RdCVFL, but not RdCVF. While intravitreal injections of 7m8-RdCVFL alone had a small, statistically insignificant effect on cone function in $r d 10$ mice, coadministration with 7m8-RdCVF resulted in higher photopic ERG amplitudes. In contrast, light-reared $r d 10$ mice that received systemic injection of AAV92YF-RdCVFL did not show improved photopic ERGs, possibly because the effect of the underlying mutation on rods is too rapid under these conditions to be rescued by expression of RdCVFL. Together, these findings suggest a role for RdCVFL in protecting the retina against oxidative stress.
Figure 6. Expression profile of $7 \mathrm{~m} 8$ after intravitreal injection at P15. (A) Fundus image of P45 WT mouse illustrating strong GFP expression across the retina after intravitreal injection of 7m8-scCAG-GFP at P15. (B) Flat mount image of a retina expressing GFP, photoreceptor-side-up, showing GFP expression in outer segments of the photoreceptors. (C) Confocal stack of transverse sections showing expression of the reporter gene across all retinal layers in a WT mouse. (D) Confocal image of a P45 rd10 retina showing GFP expression in the GCL and INL and a loss of photoreceptors resulting in loss of the ONL (arrowhead). (E) qRT-PCR indicating near-WT levels of Nxn/1 mRNA expression from eyes injected with 7m8-scCAG-RdCVF and 7m8-scCAG-RdCVFL. GFP-injected eyes had markedly reduced $N \times n / 1$ mRNA levels compared with levels in WT or AAV-injected eyes. Levels of rhodopsin mRNA were similarly low across conditions in rd10 eyes. mRNA was collected at P45 from 5 animals injected intravitreally at P15. Data represent the mean \pm SD. $n=5$ animals for each condition. Scale bars: $40 \mu \mathrm{m}$ (B); $50 \mu \mathrm{m}$ (C and D).

These results demonstrate, for the first time to our knowledge, functional photoreceptor rescue in the retina using delivery of AAV via intravascular injection. However, systemic injections may not yet be a clinically relevant method of AAV-mediated gene delivery to the retina, as this route of administration leads to widespread transduction of many tissues throughout the body, and the immune response represents a major obstacle to systemic delivery. Immune response to AAV has previously been shown to include cytotoxic T lymphocyte responses in animals with prior AAV exposure (31) and a reduction of AAV infection as a result of neutralizing antibodies (32). However, this approach illustrates the importance of early treatment in this model of rapid-progression retinal degeneration and may represent a better proof of principle for treatments using RdCVF in humans.

To examine a more clinically viable mode of delivery, we used $7 \mathrm{~m} 8$, a novel variant of AAV2, to deliver RdCVF via intravitreal injection. $7 \mathrm{~m} 8$ was selected for its ability to transduce all retinal layers, including photoreceptors, from the vitreous with near-endogenous levels of protein expression, an important aspect of the design of these experiments. The pan-retinal transduction achieved by $7 \mathrm{~m} 8$ obviates the need for penetrating subretinal injections, which require retinotomy and retinal detachment, to deliver large amounts of transgene to photoreceptors. The LCA clinical trials revealed that adverse effects, including retinal thinning and decreased visual acuity, were associated with subfoveal injections, indicating that some risk is involved with this procedure and that the human fovea is potentially vulnerable to detachment caused by a subretinal approach (33).

We found that like the systemic delivery of AAV92YF, intraocular injections of $7 \mathrm{~m} 8$ encoding RdCVF rescued cone function and prolonged cone survival, although less efficiently so, as $7 \mathrm{~m} 8$ was delivered later in the course of $r d 10$ degeneration. In patients, however, the rate of photoreceptor loss is much slower, spanning decades compared with weeks in rodent models, and therefore the window of opportunity for treatment is likely substantially extended. Finally, the rescue effects observed in $r d 10$ mice after intravitreal administration of $7 \mathrm{~m} 8$-scCAG-RdCVF were accentuated by the coadministration of $7 \mathrm{~m} 8$-scCAG-RdCVFL, suggesting the potential for a coexpression gene-therapy strategy that taps into the synergistic activities of RdCVF and RdCVFL.

In summary, these experiments support the role of Nxnl1 as a bifunctional gene encoding 2 isoforms of RdCVF with different activities in the retina. RdCVF was shown here to support cone 
A

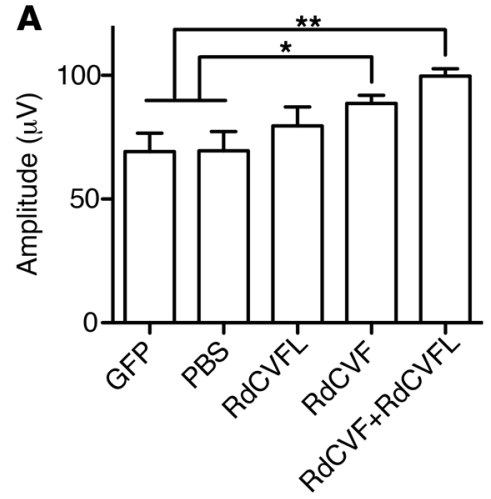

C

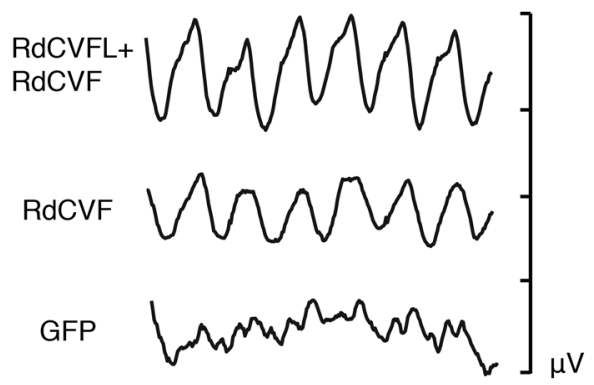

B

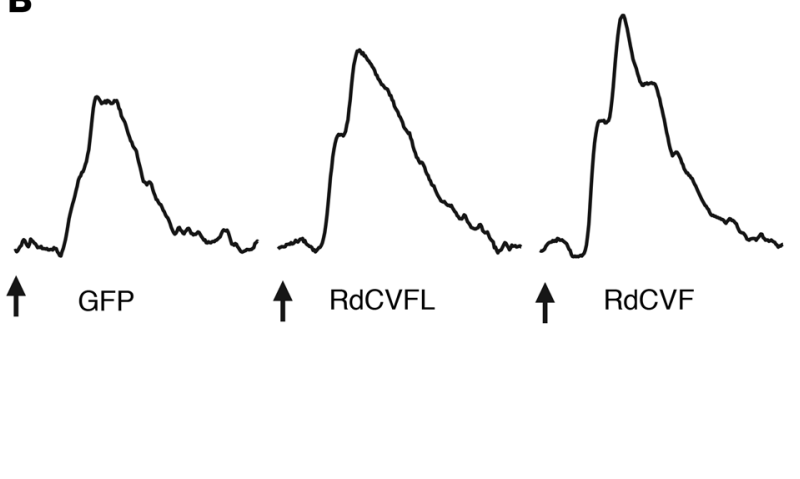

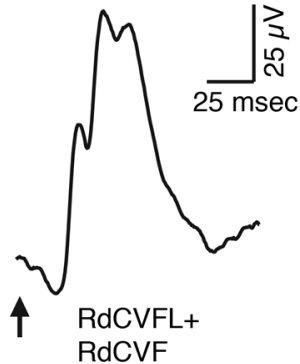

RdCVF

GFP

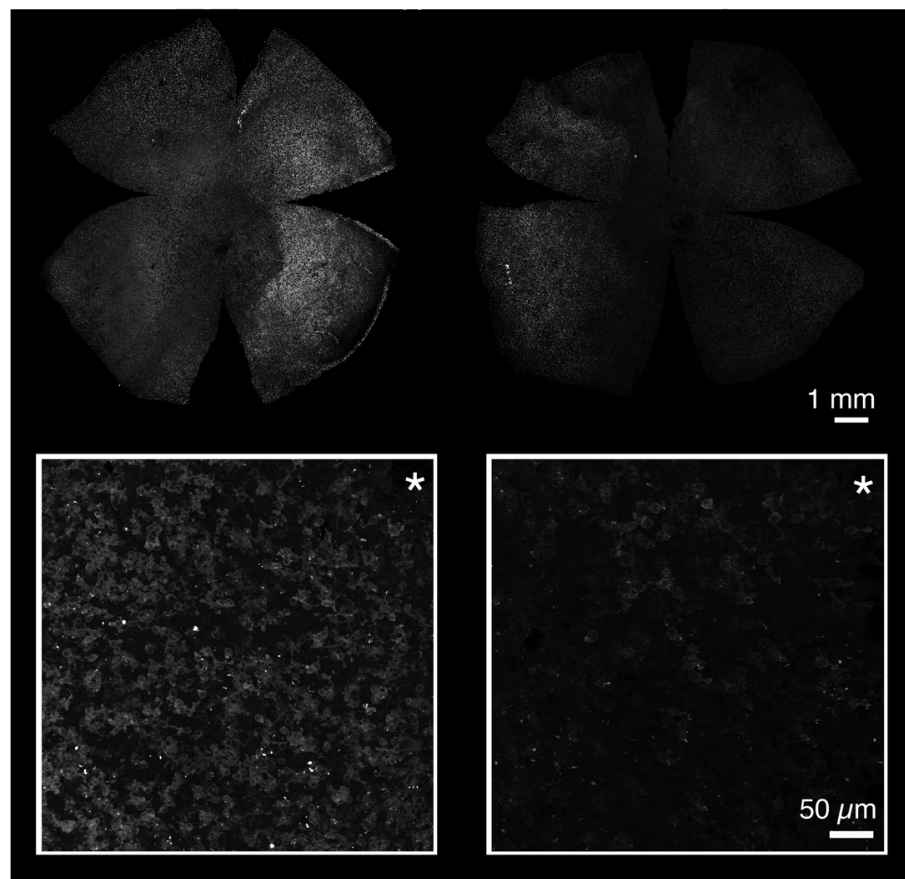

Figure 7. Intraocular injection of 7m8-scCAG-RdCVF rescues cones. (A) Photopic ERG amplitudes in rd10 animals injected at P15 with 7m8-GFP, PBS, 7m8-RdCVFL, 7m8-RdCVF, or a mix of 7m8-RdCVF plus 7m8-RdCVFL. Injection of 7m8-scCAG-RdCVFL resulted in a small but statistically insignificant increase in the amplitude of the photopic ERG b-wave compared with that measured in PBS- or GFP-injected eyes. Injection of 7m8-scCAG-RdCVF significantly increased the amplitude of the photopic ERG wave compared with that that of control eyes. Coinjection of 7m8-scCAG-RdCVF and 7m8-scCAG-RdCVFL resulted in greater rescue of the photopic ERG. $n=5$ for all groups. Significance was determined using 1-way ANOVA with Tukey's post-hoc multiple comparisons test. (B) Representative ERG traces from eyes injected with 7m8-GFP, 7m8-RdCVFL, 7m8-RdCVF, or a mixture of 7m8-RdCVF plus 7m8-RdCVFL. Arrows indicate the onset of stimulus. (C) Representative flicker ERGs recorded at $30 \mathrm{~Hz}$ of eyes injected with 7m8-RdCVF or a mix of 7m8-RdCVF plus 7m8-RdCVFL indicated improvement over ERGs of control 7m8-GFP-injected eyes. Marks on scale bar indicate $10-\mu \mathrm{V}$ increments. (D) Flat-mounted retinae labeled with anti-S-opsin and -M/L-opsin antibodies revealed higher density of cones in RdCVF-treated eyes compared with those in GFP-expressing contralateral eyes. Lower images illustrate improved densities of surviving cones in the central retina near the optic nerve head (asterisk) in 7m8-RdCVF-injected eyes. (E) Quantification of cone densities revealed greater numbers of anti-S-opsin- and anti-M/L-opsin-labeled cones in treated eyes compared with those detected in contralateral GFP-expressing eyes $(n=4)$. ${ }^{*} P<0.05$ and ${ }^{* *} P<0.01$ by 2 -tailed, paired Student's $t$ test.

survival, while RdCVFL had little direct effect on cones, but protected rod function through thioloxidoreductase activity. Further experiments are needed to understand the differing efficacies of RdCVF and RdCVFL treatment in various models of retinal degeneration, which may help us better understand the function of these isoforms in different retinal degenerative diseases. The distinct effects mediated by expression of RdCVF and RdCVFL on the survival of rods and cones in the retina support the idea that the proteins act through different mechanisms to support photoreceptors. While further studies are needed to more carefully explore the pathways underlying these rescue effects of RdCVF and RdCVFL and the benefits of their coexpression, our results indicate that viral vector-mediated delivery of the 2 products of the Nxnll gene, RdCVF and RdCVFL, represents a promising approach to treating a wide variety of retinal degenerations characterized by the loss of photoreceptors, independent of the underlying genetic cause. 
A

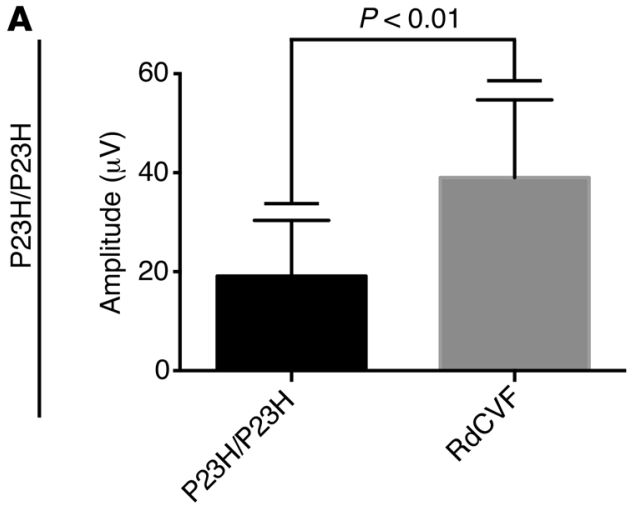

B
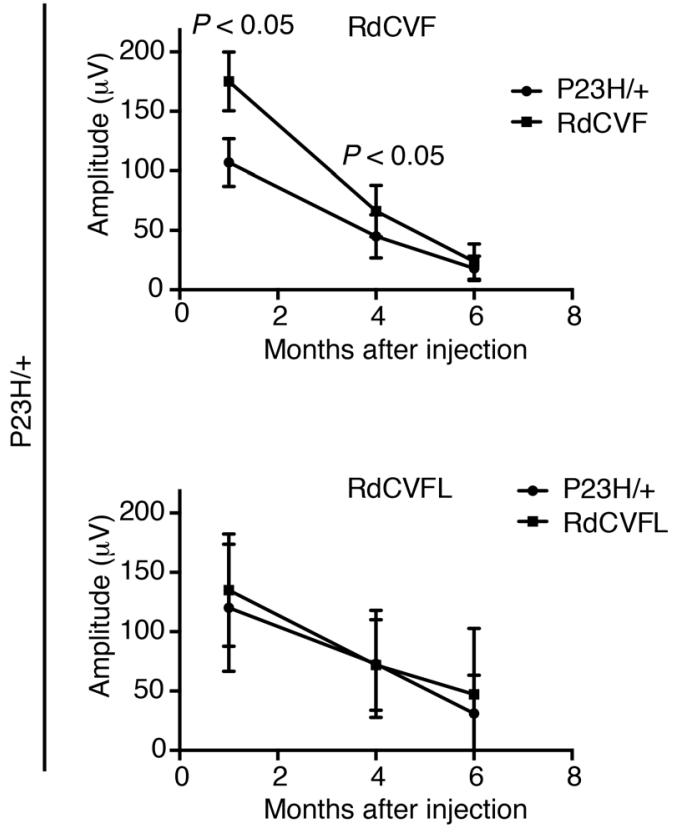

\section{Methods}

Animals. C57Bl/6J, rd10, and P23H mice were obtained from The Jackson Laboratory and raised in a 12-hour light/12-hour dark cycle unless moved to a dark box for dark rearing.

Production of viral vectors. AAV vectors carrying cDNA encoding murine RdCVF, RdCVFL, or eGFP were produced by the plasmid cotransfection method (34). Recombinant AAV was purified by iodixanol gradient ultracentrifugation and heparin column chromatography (GE Healthcare). The viral eluent was buffer exchanged and concentrated with Amicon Ultra-15 Centrifugal Filter Units (EMD Millipore) in PBS and titered by quantitative PCR relative to a standard curve.

Agarose sectioning and confocal microscopy. Retinae were freshly dissected and immediately placed in $4 \%$ paraformaldehyde overnight at $4^{\circ} \mathrm{C}$. Relief cuts were made, and whole retinae were embedded in $5 \%$ agarose. Transverse sections $(150 \mu \mathrm{m})$ were cut on a vibratome (VT 1000S; Leica Microsystems). The sections were then mounted with VECTASHIELD mounting medium (Vector Laboratories) onto slides for confocal microscopy (LSM710; Carl Zeiss).

Intravascular injections. P1 pups were immobilized, and an operating microscope was used to visualize the tail vein. Ten microliters of vector solution was drawn into a 3/10-cc insulin syringe. The 30-gauge needle was inserted into the vein, and the plunger was manually
Figure 8. Injection of 7m8-scCAG-RdCVF rescues cones in P23H mice. (A) Injections of 7m8-scCAG-RdCVF in homozygous P23H/P23H mice, a model of dominant RP, resulted in sustained amplitudes of the photopic ERG 1 month after injection $(n=6)$. (B) Time course of ERGs recorded from $\mathrm{P} 23 \mathrm{H} /+$ mice injected with 7m8-scCAG-RdCVF $(n=5)$ or $7 m 8$-scCAG-RdCVFL $(n=6)$. In mice injected with RdCVF, ERG amplitudes were higher in treated eyes compared with those in control, PBS-injected, and contralateral eyes at 1 and 4 months, but not 6 months, after injection. In mice treated with 7m8-scCAG-RdCVFL, ERG amplitudes were not significantly different from those in shaminjected eyes at any of the time points measured.

depressed. A total of $5 \times 10^{11}$ DNase-resistant particles were injected. A correct injection was verified by noting blanching of the vein. After the injection, the pups were allowed to recover for several minutes on a $37^{\circ} \mathrm{C}$ heating pad prior to being returned to their cages.

Intravitreal injections. Mice were anesthetized with ketamine $(72 \mathrm{mg} / \mathrm{kg})$ and xylazine $(64 \mathrm{mg} / \mathrm{kg})$ by i.p. injection. A 30.5-gauge disposable needle was passed through the sclera, at the equator and posterior to the limbus, into the vitreous cavity. A total of $5 \times 10^{10}$ DNase-resistant particles in a $1-\mu$ l volume was subsequently injected into the vitreous cavity with direct observation of the needle directly above the optic nerve head. Contralateral control eyes received vectors carrying the gene encoding GFP or PBS.

Dark rearing. Dark-reared mice were born and reared under dim red light in light-safe boxes, which were only opened for brief periods for animal husbandry, which was done under red light. Animals were transported to and from the box for experiments in covered cages.

$q R T-P C R$. Animals were humanely euthanized by $\mathrm{CO}_{2}$ overdose and cervical dislocation. One retina was collected from each mouse in each experimental condition $(n=5)$. RNA was extracted from each retina separately (RNeasy Micro Kit; QIAGEN) and subjected to DNase digestion, and the resulting RNA was used to create cDNA. The housekeeping gene Gapdh was used as an internal control, and no-RT controls were used to confirm the absence of genomic DNA. qRT-PCR was performed on samples using validated primers for RdCVF or rhodopsin. The following primers were used: RdCVF: forward, 5'-GAAGTGGAGACAGAGGCAGA-3'; RdCVF: reverse, 5'-AAGAAGTCTTTGAGGACTGGG-3'; rhodopsin: forward, 5'-CAAGAATCCACTGGGAGATGA-3'; rhodopsin: reverse, 5'-GTGTGTGGGGACAGGAGACT-3'; mGAPDH: forward, 5'-TGCCCCCATGTTTGTGAT-3'; and mGAPDH: reverse, 5'-TGTGGTCATGAGCCCTTCC- $3^{\prime}$. mRNA levels were determined with the relative standard curve method of qRT-PCR using a WT cDNA standard curve and are expressed as the percentage of WT mRNA levels. Individual samples were run in triplicate as technical replicates.

Fundus photography. Fundus imaging was performed with a fundus camera (Micron II; Phoenix Research Labs) equipped with a filter to monitor GFP expression in live, anesthetized mice. After application of proparacaine, pupils were dilated for fundus imaging with phenylephrine (2.5\%) and atropine sulfate (1\%).

ERGs. Mice were dark adapted for 2 hours and then anesthetized, followed by pupil dilation. Mice were placed on a $37^{\circ} \mathrm{C}$ heated pad, and contact lenses were positioned on the cornea of both eyes. A reference electrode was inserted into the forehead and a ground electrode into the tail. For an examination of retinal function under scotopic conditions, ERGs were recorded (Espion E2 ERG System; Diagnosys) under flash intensities ranging from -3 to $1 \log \mathrm{cd} \times \mathrm{s} / \mathrm{m}^{2}$ on a dark background. 
Each stimulus was presented in a series of 3 flashes. For recording of photopic ERGs, mice were initially exposed to a rod-saturating background for 10 minutes. Stimuli ranging from -0.9 to $1.4 \log \mathrm{cd} \times \mathrm{s} / \mathrm{m}^{2}$ were presented 20 times on a lighted background. Flicker ERGs were acquired following presentation of a $30-\mathrm{Hz}$ stimulus on a rod-saturating background. Stimulus intensity and timing were computer controlled. Data were analyzed with MATLAB, version 7.7 (MathWorks).

Mosaic acquisition and cone quantification. Retinal flat mounts were blocked overnight using normal donkey serum (Jackson ImmunoResearch Laboratories) 1:20 in PBS containing 0.5\% BSA, 0.1\% Triton $\mathrm{X}-100$, and $0.1 \%$ azide (PBTA) at $4^{\circ} \mathrm{C}$ and placed on a rotator for continuous agitation. Antibody cocktails containing goat anti-S-opsin (1:100; Santa Cruz Biotechnology Inc.), rabbit anti-M/ L-opsin (1:500; Chemicon International), and mouse anti-rhodopsin (1:100; gift of Robert Molday, University of British Columbia, Vancouver, British Columbia, Canada) were then added to a solution of PBTA and incubated for 2 days. Retinal preparations were then washed in PBTA 3 times for 15 minutes and 1 time for 1 hour, and subsequently corresponding secondary fluorophores were added and incubated overnight at $4^{\circ} \mathrm{C}$. Finally, samples were rinsed, mounted, and coverslipped in VECTASHIELD (Vector Labs). Images of mouse retinae were viewed and collected using an Olympus FluoView 1000 laser scanning confocal microscope with a $\times 40$ UPlanFLN (NA 1.3) oil-immersion lens. An automated stage (Applied Scientific Instrumentation) was used to capture optical sections at $1-\mu \mathrm{m}$ intervals in the $z$ axis and pixel resolution of $1,024 \times 1,024$ in the $x-y$ direction. These files were then used to create maximum-intensity projections with Imago bioimage analysis software (Mayachitra). Digital images were captured with $20 \%$ overlap among individual images, and the resulting approximately 300 to 400 images were montaged using Imago. Cone counting was subsequently performed using Imaris software (Bitplane) and custom software written in Python.

TBARS assay. MDA concentrations were determined using a TBARS assay (Cayman Chemical). Three retinae were pooled for each condition for each assay, and the assay was repeated 3 times. A total of
$25 \mathrm{mg}$ sonicated retinal tissue was used for each assay. Retinae were sonicated in lysis buffer containing a proteinase inhibitor cocktail, then centrifuged. Supernatant was used for the TBARS assay, which was performed according to the manufacturer's instructions, with technical replicates prepared in triplicate. A standard curve was prepared using MDA samples of known concentration, and sample MDA concentrations were determined against the resulting curve.

Statistics. A 1-way ANOVA with Tukey's post-hoc multiple comparisons test or a 2-tailed, paired or unpaired Student's $t$ test was used for comparisons of experimental groups. A repeated-measures 2-way ANOVA with Sidak's multiple comparisons test was used for determining statistical significance in time courses. A $P$ value of less than 0.05 was considered statistically significant and is indicated in the figures by a single asterisk. $P$ values of less than 0.01 are indicated by a double asterisk. Error bars indicate SD.

Study approval. All experiments were conducted with approval from the IACUC of the University of California Berkeley and in accordance with the Association for Research in Vision and Ophthalmology (ARVO) Statement for the Use of Animals in Ophthalmic and Vision Research and the guidelines of the Office of Laboratory Animal Care of the University of California, Berkeley.

\section{Acknowledgments}

The authors thank Miguel Betegón for help with quantification of cones. We thank Meike Visel for help with viral packaging, Jen Lam and Yvonne Lin for assistance with animal husbandry, and Trevor Lee for help with performing intraocular injections. This work was supported by NIH grants (2PN2EY018241-07), National Science Foundation Awards (0331697, III-0808772, and 0941717; UCSB), and the Foundation Fighting Blindness USA (C-GT-0711-0532-UCB03).

Address correspondence to: John G. Flannery, 112 Barker Hall, University of California, Berkeley, California 94720, USA. Phone: 510.642.0209; E-mail: flannery@berkeley.edu.
1. Buch $\mathrm{H}$, et al. Prevalence and causes of visual impairment and blindness among 9980 Scandinavian adults: the Copenhagen City Eye Study. Ophthalmology. 2004;111(1):53-61.

2. Daiger SP. RetNet: Summaries of Genes and Loci Causing Retinal Diseases. University of Texas Health Science Center Web site. https://sph.uth. edu/retnet/sum-dis.htm. Updated October 14, 2014. Accessed October 28, 2014.

3. Bramall AN, Wright AF, Jacobson SG, McInnes RR. The genomic, biochemical, and cellular responses of the retina in inherited photoreceptor degenerations and prospects for the treatment of these disorders. Annu Rev Neurosci. 2010;33(1):441-472.

4. Punzo C, Kornacker K, Cepko CL. Stimulation of the insulin/mTOR pathway delays cone death in a mouse model of retinitis pigmentosa. Nat Neurosci. 2009;12(1):44-52.

5. Léveillard T, et al. Identification and characterization of rod-derived cone viability factor. Nat Genet. 2004;36(7):755-759.

6. Mohand-Said S, et al. Normal retina releases a diffusible factor stimulating cone survival in the retinal degeneration mouse. Proc Natl Acad Sci U S A. 1998;95(14):8357-8362.

7. Mohand-Said S, et al. Photoreceptor transplants increase host cone survival in the retinal degeneration ( $\mathrm{rd}$ ) mouse. Ophthalmic Res. 1997;29(5):290-297.

8. Mohand-Said S, Hicks D, Dreyfus H, Sahel J-A. Selective transplantation of rods delays cone loss in a retinitis pigmentosa model. Arch Ophthalmol. 2000;118(6):807-811.

9. Wang XW, Tan BZ, Sun M, Ho B, Ding JL. Thioredoxin-like 6 protects retinal cell line from photooxidative damage by upregulating NF-kappaB activity. Free Radic Biol Med. 2008;45(3):336-344.

10. Yang Y, et al. Functional cone rescue by RdCVF protein in a dominant model of retinitis pigmentosa. Mol Ther. 2009;17(5):787-795.

11. Cronin $\mathrm{T}$, et al. The disruption of the rod-derived cone viability gene leads to photoreceptor dysfunction and susceptibility to oxidative stress. Cell Death Differ. 2010;17(7):1199-1210.

12. Brennan LA, Lee W, Kantorow M. TXNL6 is a novel oxidative stress-induced reducing system for methionine sulfoxide reductase a repair of $\alpha$-crystallin and cytochrome $\mathrm{C}$ in the eye lens. PLoS One. 2010;5(11):e15421.

13. Funato Y, Miki H. Nucleoredoxin, a novel thioredoxin family member involved in cell growth and differentiation. Antioxid Redox Signal. 2007;9(8):1035-1058.

14. Lillig $\mathrm{CH}$, Holmgren A. Thioredoxin and related molecules - from biology to health and disease. Antioxid Redox Signal. 2007;9(1):25-47.

15. Barhoum R, et al. Functional and structural modifications during retinal degeneration in the rd10 mouse. Neuroscience. 2008;155(3):698-713.

16. Phillips MJ, Otteson DC, Sherry DM. Progression of neuronal and synaptic remodeling in the rd10mouse model of retinitis pigmentosa. J Comp Neurol. 2010;518(11):2071-2089.

17. Gargini C, et al. Retinal organization in the retinal degeneration 10 (rd10) mutant mouse: A morphological and ERG study. J Comp Neurol. 2006;500(2):222-238.

18. Pang JJ, et al. AAV-mediated gene therapy for retinal degeneration in the rd10 mouse containing a recessive PDE $\beta$ mutation. Invest Ophthalmol Vis Sci. 
2008;49(10):4278-4283.

19. Pang JJ, et al. Long-term retinal function and structure rescue using capsid mutant AAV8 vector in the rd10 mouse, a model of recessive retinitis pigmentosa. Mol Ther. 2011;19(2):234-242.

20. Komeima K, Rogers BS, Campochiaro PA. Antioxidants slow photoreceptor cell death in mouse models of retinitis pigmentosa. JCell Physiol. 2007;213(3):809-815.

21. Sakami S, et al. Probing mechanisms of photoreceptor degeneration in a new mouse model of the common form of autosomal dominant retinitis pigmentosa due to $\mathrm{P} 23 \mathrm{H}$ opsin mutations. J Biol Chem. 2011;286(12):10551-10567.

22. Sohocki MM, et al. Prevalence of mutations causing retinitis pigmentosa and other inherited retinopathies. Hum Mutat. 2000;17(1):42-51.

23. Dalkara D, et al. In vivo-directed evolution of a new adeno-associated virus for therapeutic outer retinal gene delivery from the vitreous. Sci Transl Med. 2013;5(189):189ra76.
24. Dalkara D, et al. Enhanced gene delivery to the neonatal retina through systemic administration of tyrosine-mutated AAV9. Gene Ther. 2012;19(2):176-181.

25. Cao W, Wen R, Li F, Lavail MM, Steinberg RH. Mechanical injury increases bFGF and CNTF mRNA expression in the mouse retina. Exp Eye Res. 1997;65(2):241-248.

26. Hollander den AI, Black A, Bennett J, Cremers FPM. Lighting a candle in the dark: advances in genetics and gene therapy of recessive retinal dystrophies. JClin Invest. 2010;120(9):3042-3053.

27. Maguire AM, et al. Safety and efficacy of gene transfer for Leber's congenital amaurosis. NEngl J Med. 2008;358(21):2240-2248.

28. Cideciyan AV, et al. Human gene therapy for RPE65 isomerase deficiency activates the retinoid cycle of vision but with slow rod kinetics. Proc Natl Acad Sci U S A. 2008;105(39):15112-15117.

29. Bainbridge JWB, et al. Effect of gene therapy on visual function in Leber's congenital amaurosis.
N Engl J Med. 2008;358(21):2231-2239.

30. Fridlich R, et al. The thioredoxin-like protein rod-derived cone viability factor (RdCVFL) interacts with TAU and inhibits its phosphorylation in the retina. Mol Cell Proteomics. 2009;8(6):1206-1218.

31. Mingozzi F, et al. CD8(+) T-cell responses to adeno-associated virus capsid in humans. Nat Med. 2007;13(4):419-422.

32. Manno CS, et al. Successful transduction of liver in hemophilia by AAV-Factor IX and limitations imposed by the host immune response. Nat Med. 2006;12(3):342-347.

33. Jacobson SG, et al. Gene therapy for leber congenital amaurosis caused by RPE65 mutations: safety and efficacy in 15 children and adults followed up to 3 years. Arch Ophthalmol. 2012;130(1):9-24.

34. Grieger JC, Choi VW, Samulski RJ. Production and characterization of adeno-associated viral vectors. Nat Protoc. 2006;1(3):1412-1428. 